\title{
Metal Stents and Biliary Obstruction Secondary to Lymphoma: A Tale of Caution
}

\author{
Johan C. Bakken · Todd H. Baron
}

Published online: 8 September 2010

(C) Springer Science+Business Media, LLC 2010

We read with great interest the recent article by Ross et al. [1] reporting one cancer center's experience with the treatment of obstructive jaundice in patients with lymphoma. This article describes outcomes for 35 patients who underwent biliary decompression endoscopically or percutaneously; biliary stents were placed in 43 of the 50 ERCP's completed, and all but four of these stents were plastic.

As quoted by Ross et al. [1], the need for a permanent metal stent in cases of biliary obstruction secondary to lymphoma is less prevalent given the increased chemoresponsiveness of many lymphomas when compared to other more refractory types of malignant biliary obstruction. The authors conclude that metal stents should be reserved for patients with lymphomas refractory to treatment who are expected to live more than 6 months.

Based on experiences at our institution, we fully agree with the placement of plastic biliary stents for obstructive lymphomas, but would also urge more caution when considering uncovered metal stent placement in these cases, given the potential complications which can ensue due to the difficult (if not impossible) removal of such stents. We have two cases that illustrate this point.

The first is of a 75-year-old male who was found to have a hilar mass that was thought to be a cholangiocarcinoma or a pancreatic adenocarcinoma based on abdominal CT scan. Given the presumed poor prognosis, bilateral uncovered metal stents were placed across the bifurcation to provide bilateral intrahepatic drainage. Biopsy specimens obtained during ERCP showed diffuse large B cell lymphoma. Attempts to remove the metal stents 2 weeks

J. C. Bakken · T. H. Baron ( $₫)$

Division of Gastroenterology and Hepatology, Mayo Clinic, 200 First St SW, Rochester, MN 55905, USA

e-mail: baron.todd@mayo.edu after placement were unsuccessful due to tissue imbedding. Over the course of the next 18 months, the patient underwent six additional ERCP's to treat biliary obstruction secondary to stent occlusion from tissue hyperplasia. He ultimately underwent intrabiliary photodynamic therapy in an attempt to deliberately necrose the tissue surrounding the stents and to allow removal, but the stents could not be removed. The patient's lymphoma responded very well to chemotherapy, with no signs of recurrence, and he is still living 32 months after his diagnosis.

Our second case is of a 73-year-old female who underwent placement of an uncovered metal stent for a high-grade obstruction in the distal common bile duct thought to be secondary to pancreatic adenocarcinoma with metastases based on radiographic appearance. Ultimately, the tumor also proved to be a B cell lymphoma, and 5 months after placement, the stent was irretrievable secondary to tissue ingrowth. A covered metal stent was placed inside the original stent due to occlusion.

Uncovered metal biliary stents are nearly always nonremovable and based on our experiences, we would first and foremost recommend plastic stent placement for any confirmed hepatobiliary lymphoma resulting in biliary obstruction. For cases without confirmed tissue diagnosis, initial plastic stent placement or covered metal stent placement is an approach which may significantly limit complications arising from tissue imbedding and subsequent occlusion due to tissue hyperplasia, as our experiences have shown us.

\section{Reference}

1. Ross WA, Egwim CI, Wallace MJ, Wang M, Madoff DC, Lee JH. Outcomes in lymphoma patients with obstructive jaundice: a cancer center experience. Dig Dis Sci. 2010 July15. [Epub ahead of print]. 